\title{
Mutation based treatment recommendations from next generation sequencing data: a comparison of web tools
}

\author{
Jaymin M. Patel ${ }^{1}$, Joshua Knopf ${ }^{1}$, Eric Reiner ${ }^{3}$, Veerle Bossuyt ${ }^{2}$, Lianne Epstein ${ }^{1}$, \\ Michael DiGiovanna ${ }^{1}$, Gina Chung ${ }^{1}$, Andrea Silber ${ }^{1}$, Tara Sanft ${ }^{1}$, Erin Hofstatter ${ }^{1}$, \\ Sarah Mougalian' ${ }^{1}$, Maysa Abu-Khalaf ${ }^{1}$, James Platt ${ }^{1}$, Weiwei Shi ${ }^{1}$, Peter Gershkovich ${ }^{2}$, \\ Christos Hatzis ${ }^{1}$, Lajos Pusztai ${ }^{1}$ \\ ${ }^{1}$ Medical Oncology, Yale Cancer Center, Yale School of Medicine, CT 06520, New Haven, USA \\ ${ }^{2}$ Pathology, Yale Cancer Center, Yale School of Medicine, CT 06520, New Haven, USA \\ ${ }^{3}$ Radiology, Yale Cancer Center, Yale School of Medicine, CT 06520, New Haven, USA \\ Correspondence to: Lajos Pusztai, e-mail: lajos.pusztai@yale.edu
}

Keywords: breast cancer, biomarkers and intervention studies, mutation based treatment recommendations, tumor profiling, personalized medicine

Received: December 02, $2015 \quad$ Accepted: February 23, $2016 \quad$ Published: March 09, 2016

\begin{abstract}
Interpretation of complex cancer genome data, generated by tumor target profiling platforms, is key for the success of personalized cancer therapy. How to draw therapeutic conclusions from tumor profiling results is not standardized and may vary among commercial and academically-affiliated recommendation tools. We performed targeted sequencing of $\mathbf{3 1 5}$ genes from $\mathbf{7 5}$ metastatic breast cancer biopsies using the FoundationOne assay. Results were run through 4 different web tools including the Drug-Gene Interaction Database (DGidb), My Cancer Genome (MCG), Personalized Cancer Therapy (PCT), and cBioPortal, for drug and clinical trial recommendations. These recommendations were compared amongst each other and to those provided by FoundationOne. The identification of a gene as targetable varied across the different recommendation sources. Only $33 \%$ of cases had 4 or more sources recommend the same drug for at least one of the usually several altered genes found in tumor biopsies. These results indicate further development and standardization of broadly applicable software tools that assist in our therapeutic interpretation of genomic data is needed. Existing algorithms for data acquisition, integration and interpretation will likely need to incorporate artificial intelligence tools to improve both content and real-time status.
\end{abstract}

\section{INTRODUCTION}

Molecular target profiling of cancer is readily available in the clinic through commercial diagnostic companies and CLIA-accredited academic laboratories. Many of the assays rely on next generation sequencing methods, sometimes also including other techniques, to detect DNA sequence changes or other molecular abnormalities in tumor specimens. It is expected that the results will assist in selecting a therapy that is tailored to the specific molecular abnormalities of a given cancer [1]. Guidelines exist for the analytical validation of next generation sequencing and omics-based assays $[2,3]$ and the technical reliability of several clinically available assays have been published [4-6]. The clinical interpretation of the results, however, is less clear.
Therapeutic interpretation of genomic data from tumor biopsies is challenging at multiple levels. It has been suggested that molecular alterations in a single biopsy may not be representative of the entire tumor [7]. However, it is also clear that the majority of molecular abnormalities are shared across different regions of a cancer and even between metastatic sites, therefore the importance of private mutations is still debated. Most commercial, and many academic laboratories, perform tumor-only sequencing and it is increasingly recognized that several of the variants (up to $15-20 \%$ ) that are assumed to be somatic mutations may in fact be germline alterations. To what extent germline variants represent actionable targets is unknown [8]. Furthermore, there is no perfect method to predict the impact of a nucleic acid variant on protein function. Many computational algorithms exist that 
predict functional impact for a variant (SIFT, PolyPhen, Mutation Assessor, etc) but the true accuracy of these predictions is uncertain and they often yield conflicting results for the same variant [9]. Laboratory validation of biological impact is only available for very few mutations and variants. Finally, linking molecular abnormalities to potential therapeutic agents is also challenging and often inconsistent [10].

Putting mutations into a therapeutic context requires a broad range of expertise and a substantial amount of time on literature and database searches. Short of the handful of clinically validated targets (e.g. EGFR, c-KIT, HER-2, AKT, ROS1, BRAF, BCR-ABL, PML-RAR $\alpha$ ) that represent only a very small subset of mutated genes, there is no universal agreement, or algorithm, for determining what mutations in what genes are clinically actionable and what treatment to recommend. Many academic institutions have started molecular tumor boards which include clinical disease experts, molecular pathologists, bioinformaticians, laboratory scientists and phase I clinical trialists to assist in the clinical interpretation of complex genomic data $[11,12]$. Several academic institutions and scientific organizations have developed free online tools to assist this process. The Drug-Gene Interaction Database [13], My Cancer Genome [14], Personalized Cancer Therapy [15] and cBioPortal [16-18] represent the most readily available public resources to match genes and particular mutations to therapies that might target these. Each of these tools employs a distinct strategy that includes various combinations of automatic search algorithms and manual curation to synthesize information from databases and from the published literature.

The purpose of this study was to examine to what extent the different web tools identify similar therapeutic options for a given set of gene level anomalies observed in a cancer biopsy and if these options are concordant with those suggested by the diagnostic laboratory that has performed the molecular profiling.

\section{RESULTS}

One hundred and three genes had predicted deleterious nucleic acid variants or copy number changes in at least one of the biopsy specimens determined by a commercial diagnostic laboratory; we refer to these as "mutated genes". The median number of mutated genes per specimen was 5 (range 2 to 18). The most commonly affected gene was TP53, 37 of 75 (49\%) specimens had alterations, followed by the PIK3CA (40\%). Table 1 lists each of the affected genes and their frequencies. Forty three genes $(42 \%)$ had at least one FDA-approved drug recommendation by at least one tool (Table 3). The website that identified the most drugs was DGIdb (for 36 genes) followed by the FoundationOne report (for 31 genes), cBioPortal (for 25 genes), MCG (10 genes) and PCT (for 9 genes). The most commonly recommended drugs were temsirolimus for alterations in 15 genes (PIK3CA, PIK3R1, PTEN, STK11, AKT-1, -2, -3, TSC2, KIT, PDGFRB, KRAS, VHL, RPTOR, NF1, BRAF) followed by everolimus for 14 genes (same as temsirolimus except BRAF) and regorafenib for 10 genes (FGFR -1, -2, FLT -2, -4, KDR, KIT, KRAS, PDGFR $\beta$, VHL, BRAF) (Table 3).

However, for a given mutated gene, a drug recommendation by one source did not imply identical recommendation from another source. Only for 2 genes (KIT, FLT3) did all 5 sources recommended the same drug. Three other genes (BRAF, FGFR1, FGFR2,) had drug recommendation from all 5 sources, but the recommendations were partially discordant (Figure 1 and Table 2). There were 7 genes (PDGFRB, BRAF, EGFR, ERBB2, FGFR1, SRC \& FGFR2) with the same drug recommendation from 4 sources and 11 genes with drug recommendations from 3 sources; among these 10 had at least one drug recommended by all 3 sources. There were 12 genes for which only 2 web sites made drug recommendations, but 11 of these genes had the same drug recommended by both. These results indicate only partial overlap in identifying similar treatment options based on the same mutation data among different web-based tools and FoundationOne.

The median numbers of drugs for a targetable gene identified by the different tools was 2 for Foundation One (range 1-11), 3 for DGIdb (range: 1-24), 5 for PCT (range: 1-10), 2 for MCG (range 1-7) and 2 for cBioPortal (range: 1-20). Clinical trial recommendations were reported for 56 of the 103 genes by FoundationOne. Only 7 of these genes (AKT1, BRAF, FGFR1, FGFR2, KIT, PIK3CA \& PTEN) had at least one clinical trial that was recommended by both the FoundationOne report and another source (Figure 2). The median number of clinical trials recommended by FoundationOne (for 56 genes), MCG (for 5 genes) and PCT (for 9 genes) was 2, 51 and 9 respectively.

For individual cases, the drug or clinical trial recommendations ranged from high degree of overlap to no overlap across the 5 tools. Figure 3 illustrates recommendations at the cohort level, case level and gene level for a single case. This cancer had 10 mutated genes with multiple drug recommendations that illustrate the various degrees of overlap in treatment options. For example, KIT had identical recommendations by all 5 tools, KRAS, TOP1 and JAK2 had 3 tools reporting the same drug as an option, while GNAS and TP53 each had drug recommendations by FoundationOne only. Alterations in AURKA, BRCA2, SMAD4 \& ZNF217 had no drug recommendations. Overall, 3 cases had at least 1 gene with an identical drug recommendation from all 5 sources, 22 cases with an overlapping recommendation from 4 sources, 32 cases from 3 sources, 12 cases from 2 sources and 6 with no overlapping recommendations at all. Additionally, 21 cases had at least 1 overlapping clinical trial recommendation from 3 sources, 33 cases had 2 sources and 21 cases had at least 1 gene 
Table 1: Mutations from 75 advanced breast cancer cases in order of frequency

\begin{tabular}{|c|c|c|c|c|c|c|c|c|}
\hline Gene & $\%$ Cases & \# Cases & Gene & $\%$ Cases & \# Cases & Gene & $\%$ Cases & \# Cases \\
\hline TP53 & $49.33 \%$ & 37 & ERBB3 & $4.00 \%$ & 3 & CARD11 & $1.33 \%$ & 1 \\
\hline PIK3CA & $40.00 \%$ & 30 & LRP1B & $4.00 \%$ & 3 & CDK4 & $1.33 \%$ & 1 \\
\hline CCND1 & $30.67 \%$ & 23 & MDM4 & $4.00 \%$ & 3 & CTNNB1 & $1.33 \%$ & 1 \\
\hline FGF19 & $30.67 \%$ & 23 & MLL2 & $4.00 \%$ & 3 & EGFR & $1.33 \%$ & 1 \\
\hline FGF3 & $30.67 \%$ & 23 & RAD50 & $4.00 \%$ & 3 & ETV6 & $1.33 \%$ & 1 \\
\hline FGF4 & $30.67 \%$ & 23 & RB1 & $4.00 \%$ & 3 & FANCA & $1.33 \%$ & 1 \\
\hline ESR1 & $26.67 \%$ & 20 & RUNX1 & $4.00 \%$ & 3 & FGFR4 & $1.33 \%$ & 1 \\
\hline MYC & $24.00 \%$ & 18 & ATR & $2.67 \%$ & 2 & $\underline{\text { FLT3 }}$ & $1.33 \%$ & 1 \\
\hline $\mathrm{CDH} 1$ & $18.67 \%$ & 14 & CCND2 & $2.67 \%$ & 2 & FLT4 & $1.33 \%$ & 1 \\
\hline PTEN & $18.67 \%$ & 14 & CREBBP & $2.67 \%$ & 2 & HRAS & $1.33 \%$ & 1 \\
\hline EMSY & $14.67 \%$ & 11 & EPHB1 & $2.67 \%$ & 2 & JAK2 & $1.33 \%$ & 1 \\
\hline ZNF217 & $14.67 \%$ & 11 & FGF14 & $2.67 \%$ & 2 & KDM5A & $1.33 \%$ & 1 \\
\hline GATA3 & $12.00 \%$ & 9 & FGF23 & $2.67 \%$ & 2 & МАР2К2 & $1.33 \%$ & 1 \\
\hline MYST3 & $12.00 \%$ & 9 & FGF6 & $2.67 \%$ & 2 & MEN1 & $1.33 \%$ & 1 \\
\hline FGFR1 & $10.67 \%$ & 8 & IKBKE & $2.67 \%$ & 2 & MITF & $1.33 \%$ & 1 \\
\hline ARFRP1 & $9.33 \%$ & 7 & JUN & $2.67 \%$ & 2 & MSH2 & $1.33 \%$ & 1 \\
\hline MAP2K4 & $9.33 \%$ & 7 & KDR & $2.67 \%$ & 2 & MYCL1 & $1.33 \%$ & 1 \\
\hline TOP1 & $9.33 \%$ & 7 & $\underline{\text { KIT }}$ & $2.67 \%$ & 2 & MYCN & $1.33 \%$ & 1 \\
\hline CCNE1 & $8.00 \%$ & 6 & MCL1 & $2.67 \%$ & 2 & NF1 & $1.33 \%$ & 1 \\
\hline$\underline{\text { FGFR2 }}$ & $8.00 \%$ & 6 & NOTCH1 & $2.67 \%$ & 2 & NFKBIA & $1.33 \%$ & 1 \\
\hline MAP3K1 & $8.00 \%$ & 6 & PDGFRB & $2.67 \%$ & 2 & NKX2-1 & $1.33 \%$ & 1 \\
\hline AURKA & $6.67 \%$ & 5 & RPTOR & $2.67 \%$ & 2 & PALB2 & $1.33 \%$ & 1 \\
\hline CDKN2A & $6.67 \%$ & 5 & SPEN & $2.67 \%$ & 2 & PAX5 & $1.33 \%$ & 1 \\
\hline CDKN2B & $6.67 \%$ & 5 & STK11 & $2.67 \%$ & 2 & PIK3R1 & $1.33 \%$ & 1 \\
\hline ERBB2 & $6.67 \%$ & 5 & ZNF703 & $2.67 \%$ & 2 & PRKDC & $1.33 \%$ & 1 \\
\hline MDM2 & $6.67 \%$ & 5 & $\underline{\mathrm{AKT} 1}$ & $1.33 \%$ & 1 & RARA & $1.33 \%$ & 1 \\
\hline ARID1A & $5.33 \%$ & 4 & AKT2 & $1.33 \%$ & 1 & SMAD4 & $1.33 \%$ & 1 \\
\hline ATM & $5.33 \%$ & 4 & AKT3 & $1.33 \%$ & 1 & SMARCA4 & $1.33 \%$ & 1 \\
\hline CCND3 & $5.33 \%$ & 4 & APC & $1.33 \%$ & 1 & SRC & $1.33 \%$ & 1 \\
\hline GNAS & $5.33 \%$ & 4 & ARID2 & $1.33 \%$ & 1 & SUFU & $1.33 \%$ & 1 \\
\hline IGF1R & $5.33 \%$ & 4 & ATRX & $1.33 \%$ & 1 & TET2 & $1.33 \%$ & 1 \\
\hline$\underline{\text { KRAS }}$ & $5.33 \%$ & 4 & BCL2L2 & $1.33 \%$ & 1 & TSC2 & $1.33 \%$ & 1 \\
\hline SOX2 & $5.33 \%$ & 4 & $\underline{\text { BRAF }}$ & $1.33 \%$ & 1 & VHL & $1.33 \%$ & 1 \\
\hline BRCA2 & $4.00 \%$ & 3 & BRIP1 & $1.33 \%$ & 1 & & & \\
\hline CDK12 & $4.00 \%$ & 3 & BTK & $1.33 \%$ & 1 & & & \\
\hline
\end{tabular}

Note: Genes in bold have at least 1 FDA approved drug recommendation. Genes with underline have at least 1 clinical trial recommendation from multiple sources. 
Table 2: FDA approved drug recommendations listed by mutation and source

\begin{tabular}{|c|c|c|c|c|c|}
\hline Gene & Foundation & DGIdb & MCG & PCT & cBioPortal \\
\hline AKT1 & $\begin{array}{l}\text { Everolimus, } \\
\text { Temsirolimus }\end{array}$ & $\begin{array}{c}\text { Everolimus, Temsirolimus, } \\
\text { Nelfinavir, } \\
\text { Risperidone }\end{array}$ & & $\begin{array}{l}\text { Everolimus, } \\
\text { Temsirolimus, } \\
\text { Metformin, } \\
\text { Sirolimus } \\
\end{array}$ & Arsenic Trioxide \\
\hline AKT2 & $\begin{array}{l}\text { Everolimus, } \\
\text { Temsirolimus }\end{array}$ & Everolimus & & & \\
\hline AKT3 & $\begin{array}{l}\text { Everolimus, } \\
\text { Temsirolimus }\end{array}$ & Everolimus & & & \\
\hline ATR & & Olaparib & & & \\
\hline BRAF & $\begin{array}{l}\text { Sorafenib, } \\
\text { Trametinib, } \\
\text { Regorafenib }\end{array}$ & $\begin{array}{c}\text { Sorafenib, Dabrafenib, } \\
\text { Vemurafenib, Regorafenib, } \\
\text { Trametinib, Dasatinib, } \\
\text { Temsirolimus }\end{array}$ & $\begin{array}{l}\text { Dabrafenib, } \\
\text { Vemurafenib, } \\
\text { Trametinib }\end{array}$ & $\begin{array}{c}\text { Sorafenib, } \\
\text { Dabrafenib, } \\
\text { Vemurafenib, } \\
\text { Regorafenib, } \\
\text { Trametinib }\end{array}$ & $\begin{array}{c}\text { Sorafenib, Dabrafenib, } \\
\text { Vemurafenib }\end{array}$ \\
\hline BTK & & Ibrutinib & Ibrutinib & & Ibrutinib \\
\hline CCND1 & & $\begin{array}{l}\text { Arsenic Trioxide, } \\
\text { Methotrexate }\end{array}$ & & & Arsenic Trioxide \\
\hline CTNNB1 & & Celecoxib & & & \\
\hline EGFR & $\begin{array}{c}\text { Afatinib, Erlotinib, } \\
\text { Gefitinib, } \\
\text { Cetuximab, } \\
\text { Lapatinib, } \\
\text { Panitumumab }\end{array}$ & $\begin{array}{c}\text { Afatinib, Erlotinib, } \\
\text { Gefitinib, Cetuximab, } \\
\text { Lapatinib, Panitumumab, } \\
\text { Vandetanib, Lidocaine, } \\
\text { Trastuzumab, Bevacizumab, } \\
\text { Pazopanib, Pertuzumab }\end{array}$ & $\begin{array}{l}\text { Afatinib, } \\
\text { Erlotinib, } \\
\text { Gefitinib, } \\
\text { Vandetanib }\end{array}$ & & $\begin{array}{c}\text { Afatinib, Erlotinib, } \\
\text { Gefitinib, Cetuximab, } \\
\text { Lapatinib, } \\
\text { Panitumumab, } \\
\text { Vandetanib, Lidocaine, } \\
\text { Trastuzumab } \\
\end{array}$ \\
\hline ERBB2 & $\begin{array}{l}\text { Afatinib, Lapatinib, } \\
\text { Pertuzumab, } \\
\text { Trastuzumab, Ado- } \\
\text { trastuzumab }\end{array}$ & $\begin{array}{c}\text { Afatinib, Lapatinib, } \\
\text { Pertuzumab, Trastuzumab, } \\
\text { Ado-Trastuzumab, } \\
\text { Bevacizumab, } \\
\text { Gefitinib }\end{array}$ & $\begin{array}{l}\text { Afatinib, } \\
\text { Lapatinib }\end{array}$ & & $\begin{array}{l}\text { Afatinib, Lapatinib, } \\
\text { Pertuzumab, } \\
\text { Trastuzumab }\end{array}$ \\
\hline ERBB3 & $\begin{array}{c}\text { Afatinib, } \\
\text { Pertuzumab }\end{array}$ & Gefitinib & & & \\
\hline ESR1 & & $\begin{array}{c}\text { Clomiphene, Danazol, } \\
\text { Desogestrel, Estramustine, } \\
\text { Estropipate, Ethinyl } \\
\text { Estradiol, } \\
\text { Ethynodiol Diacetate, } \\
\text { Fluoxymesterone, } \\
\text { Fulvestrant, Levonorgestrel, } \\
\text { Medroxyprogesterone, } \\
\text { Megestrol, Naloxone, } \\
\text { Norgestimate, Norgestrel, } \\
\text { Progesterone, Raloxifene, } \\
\text { Tamoxifen, Toremifene, } \\
\text { Anastrozole, Cisplatin, } \\
\text { Exemestane, Leflunomide, } \\
\text { Letrozole }\end{array}$ & & & $\begin{array}{c}\text { Clomiphene, Danazol, } \\
\text { Desogestrel, } \\
\text { Estramustine, } \\
\text { Estropipate, Ethinyl } \\
\text { Estradiol } \\
\text { Ethynodiol Diacetate, } \\
\text { Fluoxymesterone, } \\
\text { Fulvestrant, } \\
\text { Levonorgestrel, } \\
\text { Medroxyprogesterone, } \\
\text { Megestrol, Naloxone, } \\
\text { Norgestimate, } \\
\text { Norgestrel, } \\
\text { Progesterone, } \\
\text { Raloxifene, Tamoxifen, } \\
\text { Toremifene, } \\
\text { Bazedoxifene }\end{array}$ \\
\hline FGF4 & & Pentosan Polysulfate & & & Pentosan Polysulfate \\
\hline
\end{tabular}




\begin{tabular}{|c|c|c|c|c|c|}
\hline FGFR1 & $\begin{array}{c}\text { Regorafenib, } \\
\text { Ponatinib, } \\
\text { Pazopanib }\end{array}$ & $\begin{array}{c}\text { Regorafenib, } \\
\text { Ponatinib, Palifermin }\end{array}$ & $\begin{array}{l}\text { Ponatinib, } \\
\text { Nintedanib }\end{array}$ & $\begin{array}{l}\text { Regorafenib, } \\
\text { Ponatinib, } \\
\text { Pazopanib, } \\
\text { Dasatinib, } \\
\text { Sorafenib, } \\
\text { Sunitinib, } \\
\text { Vandetanib } \\
\end{array}$ & $\begin{array}{c}\text { Regorafenib, } \\
\text { Palifermin }\end{array}$ \\
\hline FGFR2 & $\begin{array}{l}\text { Ponatinib, } \\
\text { Pazopanib }\end{array}$ & $\begin{array}{l}\text { Ponatinib, Palifermin, } \\
\text { Regorafenib, Thalidomide, } \\
\text { Interferon, Pentosan } \\
\text { Polysulfate }\end{array}$ & $\begin{array}{l}\text { Ponatinib, } \\
\text { Nintedanib }\end{array}$ & $\begin{array}{l}\text { Ponatinib, } \\
\text { Pazopanib, } \\
\text { Regorafenib, } \\
\text { Sorafenib, } \\
\text { Sunitinib } \\
\end{array}$ & $\begin{array}{l}\text { Palifermin, } \\
\text { Thalidomide }\end{array}$ \\
\hline FGFR4 & Ponatinib & Palifermin & & & Palifermin \\
\hline FLT3 & $\begin{array}{c}\text { Sorafenib, } \\
\text { Sunitinib, Ponatinib }\end{array}$ & $\begin{array}{c}\text { Sorafenib, Sunitinib, } \\
\text { Ponatinib, Cabozantinib, } \\
\text { Azacitidine, Bortezomib, } \\
\text { Clofarabine, Idarubicin }\end{array}$ & $\begin{array}{c}\text { Sorafenib, } \\
\text { Sunitinib, } \\
\text { Ponatinib, } \\
\text { Cabozantinib }\end{array}$ & $\begin{array}{l}\text { Sorafenib, } \\
\text { Sunitinib, } \\
\text { Ponatinib, } \\
\text { Cabozantinib, } \\
\text { Vandetanib, } \\
\text { Pazopanib, } \\
\text { Regorafenib } \\
\end{array}$ & $\begin{array}{l}\text { Sorafenib, Sunitinib, } \\
\text { Vandetanib }\end{array}$ \\
\hline FLT4 & $\begin{array}{l}\text { Axitinib, } \\
\text { Pazopanib, } \\
\text { Regorafenib, } \\
\text { Sorafenib, } \\
\text { Sunitinib, } \\
\text { Vandetanib } \\
\end{array}$ & $\begin{array}{l}\text { Axitinib, Pazopanib, } \\
\text { Regorafenib, Sorafenib, } \\
\text { Sunitinib, Vandetanib }\end{array}$ & & & $\begin{array}{c}\text { Axitinib, Pazopanib, } \\
\text { Regorafenib, Sorafenib, } \\
\text { Sunitinib, Vandetanib, } \\
\text { Cabozantinib }\end{array}$ \\
\hline GNAS & Trametinib & & & & \\
\hline HRAS & Trametinib & & & & \\
\hline IGF1R & & $\begin{array}{l}\text { Glargine Insulin, Lispro } \\
\text { Insulin, Porcine Insulin }\end{array}$ & & & $\begin{array}{c}\text { Glargine Insulin, } \\
\text { Lispro Insulin, Porcine } \\
\text { Insulin, Mecasermin } \\
\end{array}$ \\
\hline JAK2 & & Ruxolitinib & Ruxolitinib & & Ruxolitinib \\
\hline JUN & & $\begin{array}{c}\text { Arsenic Trioxide, Irbesartan, } \\
\text { Vinblastine }\end{array}$ & & & $\begin{array}{c}\text { Arsenic Trioxide, } \\
\text { Irbesartan, Vinblastine }\end{array}$ \\
\hline KDR & $\begin{array}{l}\text { Pazopanib, } \\
\text { Sorafenib, } \\
\text { Sunitinib, } \\
\text { Vandetanib, } \\
\text { Axitinib, } \\
\text { Regorafenib, } \\
\text { Bevacizumab }\end{array}$ & $\begin{array}{c}\text { Pazopanib, Sorafenib, } \\
\text { Sunitinib, Vandetanib, } \\
\text { Cabozantinib, } \\
\text { Ramucirumab, Regorafenib, } \\
\text { Ponatinib }\end{array}$ & & & $\begin{array}{c}\text { Pazopanib, Sorafenib, } \\
\text { Sunitinib, Vandetanib, } \\
\text { Axitinib, Cabozantinib, } \\
\text { Ramucirumab }\end{array}$ \\
\hline KIT & \begin{tabular}{|} 
Dasatinib, Imatinib, \\
Pazopanib, \\
Regorafenib, \\
Sunitinib, Nilotinib, \\
Sorafenib, \\
Ponatinib, \\
Everolimus, \\
Temsirolimus
\end{tabular} & $\begin{array}{l}\text { Dasatinib, Imatinib, } \\
\text { Pazopanib, Regorafenib, } \\
\text { Sunitinib, Axitinib, } \\
\text { Nilotinib, Sorafenib, } \\
\text { Cabozantinib }\end{array}$ & $\begin{array}{l}\text { Dasatinib, } \\
\text { Imatinib, } \\
\text { Pazopanib, } \\
\text { Regorafenib, } \\
\text { Sunitinib, } \\
\text { Axitinib, } \\
\text { Cabozantinib }\end{array}$ & $\begin{array}{l}\text { Dasatinib, } \\
\text { Imatinib, } \\
\text { Pazopanib, } \\
\text { Regorafenib, } \\
\text { Sunitinib, } \\
\text { Axitinib, } \\
\text { Nilotinib, } \\
\text { Sorafenib, } \\
\text { Cabozantinib, } \\
\text { Pazopanib, } \\
\text { Ponatinib }\end{array}$ & $\begin{array}{c}\text { Dasatinib, Imatinib, } \\
\text { Pazopanib, } \\
\text { Regorafenib, } \\
\text { Sunitinib, Axitinib, } \\
\text { Nilotinib, Sorafenib, } \\
\text { Cabozantinib, } \\
\text { Vandetanib }\end{array}$ \\
\hline
\end{tabular}




\begin{tabular}{|c|c|c|c|c|c|}
\hline KRAS & Trametinib & $\begin{array}{c}\text { Trametinib, Cetuximab, } \\
\text { Everolimus, Erlotinib, } \\
\text { Gefitinib, Panitumumab, } \\
\text { Regorafenib, Simvastatin, } \\
\text { Temsirolimus, } \\
\text { Vandetanib }\end{array}$ & & Trametinib & \\
\hline MAP2K2 & Trametinib & Trametinib, Dabrafenib & & & Trametinib \\
\hline МАР2K4 & & Trametinib, Dabrafenib & & & \\
\hline NF1 & $\begin{array}{c}\text { Everolimus, } \\
\text { Temsirolimus, } \\
\text { Trametinib }\end{array}$ & Trametinib & & & \\
\hline PDGFRB & $\begin{array}{c}\text { Imatinib, } \\
\text { Pazopanib, } \\
\text { Regorafenib, } \\
\text { Sorafenib, } \\
\text { Sunitinib, } \\
\text { Dasatinib, } \\
\text { Everolimus, } \\
\text { Ponatinib, } \\
\text { Temsirolimus }\end{array}$ & $\begin{array}{l}\text { Imatinib, Pazopanib, } \\
\text { Regorafenib, } \\
\text { Sorafenib, Sunitinib, } \\
\text { Axitinib, Dasatinib, } \\
\text { Nilotinib }\end{array}$ & $\begin{array}{c}\text { Imatinib, } \\
\text { Pazopanib, } \\
\text { Regorafenib, } \\
\text { Sorafenib, } \\
\text { Sunitinib, } \\
\text { Axitinib, } \\
\text { Nintedanib }\end{array}$ & & $\begin{array}{c}\text { Imatinib, Pazopanib, } \\
\text { Regorafenib, } \\
\text { Sorafenib, Sunitinib, } \\
\text { Axitinib, Dasatinib, } \\
\text { Nilotinib, } \\
\text { Cabozantinib }\end{array}$ \\
\hline PIK3CA & $\begin{array}{l}\text { Everolimus, } \\
\text { Temsirolimus }\end{array}$ & $\begin{array}{c}\text { Everolimus, Temsirolimus, } \\
\text { Docetaxel }\end{array}$ & & $\begin{array}{c}\text { Everolimus, } \\
\text { Temsirolimus, } \\
\text { Sirolimus, } \\
\text { Metformin }\end{array}$ & \\
\hline PIK3R1 & $\begin{array}{l}\text { Everolimus, } \\
\text { Temsirolimus }\end{array}$ & Isoproterenol & & & Isoproterenol \\
\hline PTEN & $\begin{array}{c}\text { Everolimus, } \\
\text { Temsirolimus }\end{array}$ & $\begin{array}{c}\text { Everolimus, Temsirolimus, } \\
\text { Cetuximab, Erlotinib, } \\
\text { Gefitinib, Panitumumab, } \\
\text { Vandetanib }\end{array}$ & & $\begin{array}{c}\text { Everolimus, } \\
\text { Temsirolimus, } \\
\text { Sirolimus }\end{array}$ & \\
\hline RARA & & $\begin{array}{c}\text { Acitretin, Etretinate, } \\
\text { Isotretinoin, Arsenic } \\
\text { Trioxide, Tretinoin }\end{array}$ & & & $\begin{array}{l}\text { Acitretin, Etretinate, } \\
\text { Isotretinoin }\end{array}$ \\
\hline RB1 & & $\begin{array}{c}\text { Porcine Insulin, } \\
\text { Recombinant Insulin }\end{array}$ & & & $\begin{array}{c}\text { Porcine Insulin, } \\
\text { Recombinant Insulin }\end{array}$ \\
\hline RPTOR & $\begin{array}{l}\text { Everolimus, } \\
\text { Temsirolimus }\end{array}$ & Sirolimus, Temsirolimus & & & \\
\hline SRC & $\begin{array}{l}\text { Dasatinib, } \\
\text { Bosutinib }\end{array}$ & $\begin{array}{c}\text { Dasatinib, Bosutinib, } \\
\text { Ponatinib }\end{array}$ & Dasatinib & & Dasatinib, Bosutinib \\
\hline STK11 & $\begin{array}{l}\text { Everolimus, } \\
\text { Temsirolimus }\end{array}$ & & & & \\
\hline TET2 & $\begin{array}{l}\text { Azacitidine, } \\
\text { Decitabine }\end{array}$ & & & & \\
\hline TOP1 & $\begin{array}{l}\text { Irinotecan, } \\
\text { Topotecan }\end{array}$ & Irinotecan, Topotecan & & & Irinotecan, Topotecan \\
\hline TP53 & & Fluorouracil, Paclitaxel & & & \\
\hline TSC2 & $\begin{array}{l}\text { Everolimus, } \\
\text { Temsirolimus }\end{array}$ & & & & \\
\hline
\end{tabular}




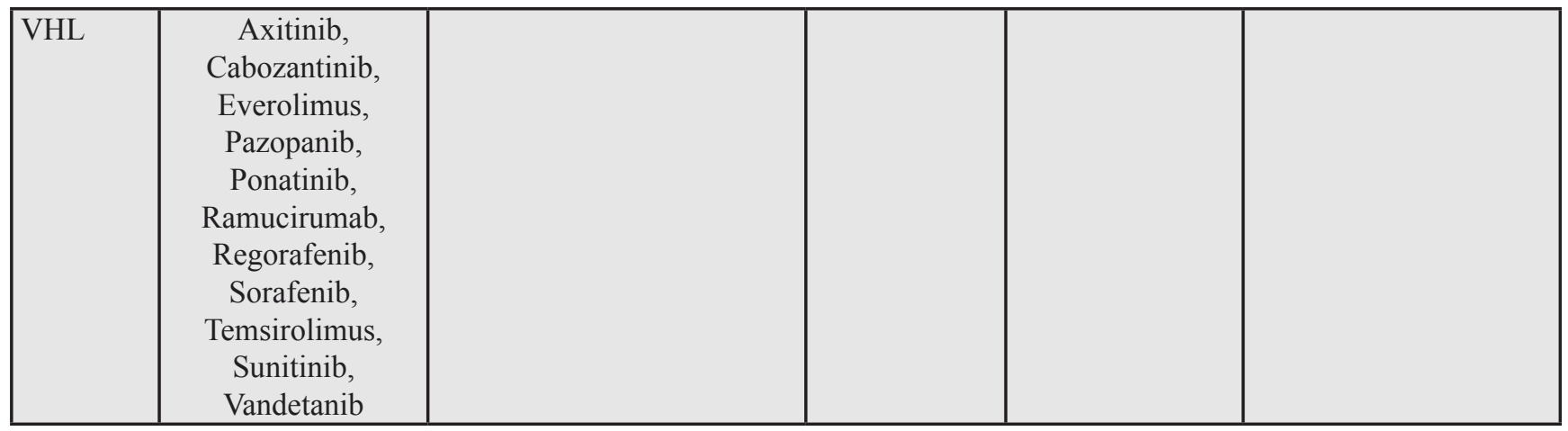

Note: Drugs with overlap in recommendation sources are listed in bold for four-way overlap and in bold + underline for five-way overlap.

with a clinical trial recommendation from FoundationOne, but no recommendations from any of the other sources. In summary, $33 \%$ of the metastatic breast cancer cases had a drug recommendation for at least 1 of its mutated genes that was agreed upon by 4 or more of the sources and $72 \%$ had a clinical trial recommendation agreed upon by 2 or more of the sources.

\section{DISCUSSION}

The purpose of this study was to examine to what extent different web tools and a widely used commercial service identify similar therapeutic options for a given set of genomic anomalies in a cancer. Our data represents the typical clinical scenario of tumor only

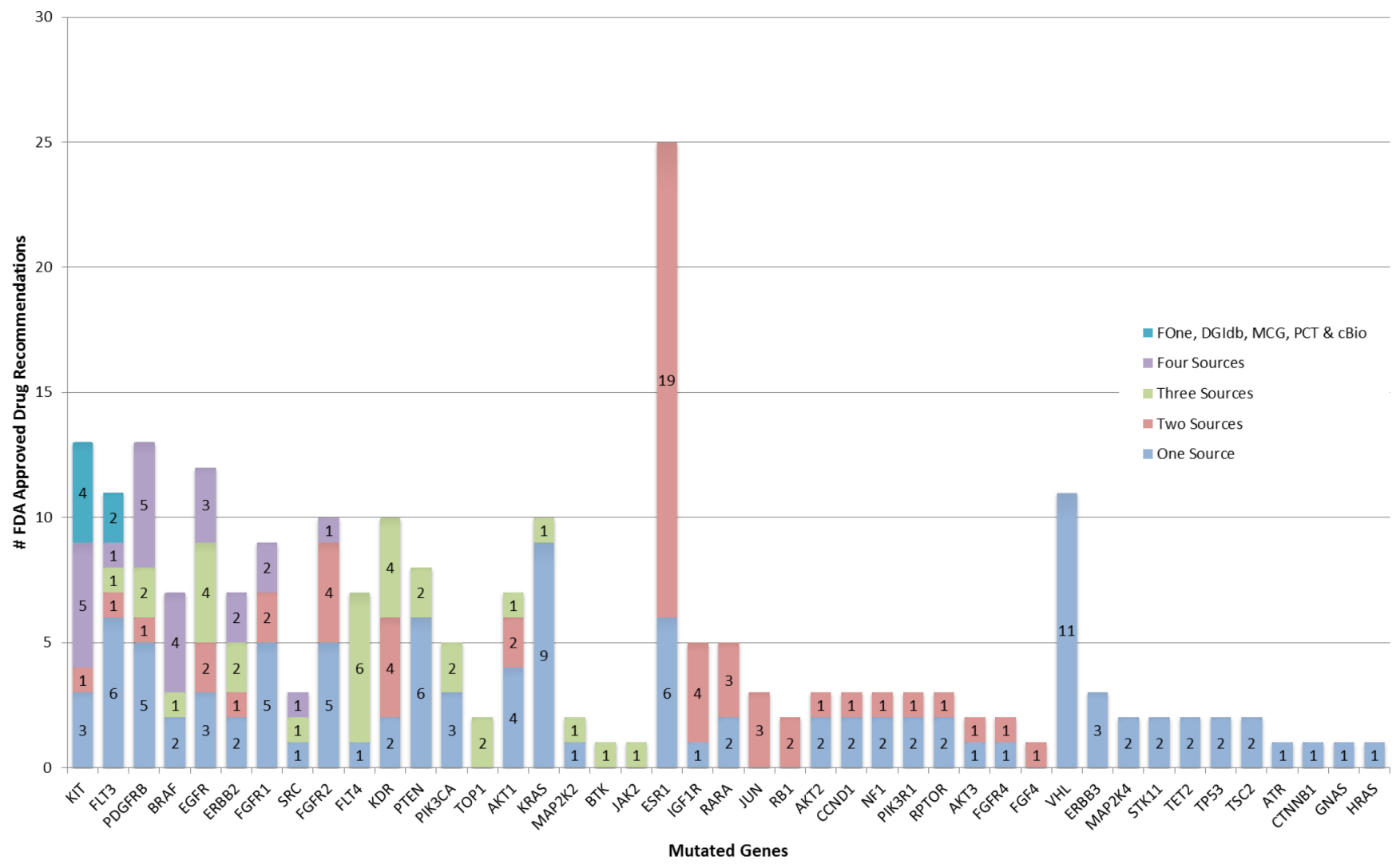

Figure 1: Occurrence of FDA approved drug recommendations. Mutated genes from highest to lowest number of sources are listed along $\mathrm{x}$-axis and number of drug recommendations grouped by number of contributing sources are stacked along $\mathrm{y}$-axis. 
research biopsies obtained mostly from a metastatic site in heavily pretreated patients. Target profiling was performed by FoundationOne assay. We observed only partial overlap in finding similar treatment options based on the same mutation data among 5 recommendation sources designed to enable personalized medicine. Only $33 \%$ of cases had a drug recommendation agreed upon by 4 or more sources for at least 1 mutated gene. The different tools identified different number of genes as targetable with off-label use of FDA-approved drugs. Using the same input data from the FoundationOne reports, DGIdb identified 36 genes, FoundationOne identified 31 genes, cBioPortal 25 genes, MCG 10 genes and PCT 9 genes as potentially targetable. Furthermore, clinical trial recommendations were provided by only half of the web tools (MCG \& PCT) and were less frequently provided for both web tools combined (14 genes) than FoundationOne alone (56 genes). While we recognize that identical treatment recommendations for a given molecular abnormality from multiple sources does not imply clinical validity or increase the likelihood that the suggested treatment would work, our findings highlight the challenges in interpreting clinical tumor profiling results.
Different therapeutic conclusions can be drawn from the same data depending on what analytic tool is used. This is primarily due to the different rules used by different tools to define what constitutes a druggable gene. Concordance was greater when there was high level of evidence from clinical trials that a given drug directly targets a particular molecular abnormality (e.g. Kit, BRAF, ERBB2). However, for the majority of mutations and affected genes no such drug exists. In these instances, potential drug efficacy is inferred from biological principles or from off-target effects of kinase inhibitors. Different websites apply different rules to such circumstantial evidence that largely explains the substantial discordance. For example, the most frequently, but not the most concordantly recommended drug, was the mTOR inhibitor temsirolimus for alterations in PIK3CA, PIK3R1, PTEN, STK11, AKT-1, -2-, 3, TSC2, KIT, PDGFRB, KRAS, VHL, RPTOR, NF1 and BRAF based on biological pathway-level association. The effect of temsirolimus on these targets is hypothetical since no mutations in any of these genes have been directly linked to temsirolimus sensitivity in patients, therefore discordant recommendations are not unexpected. A more concerning example is FGFR4 amplification. One source identified

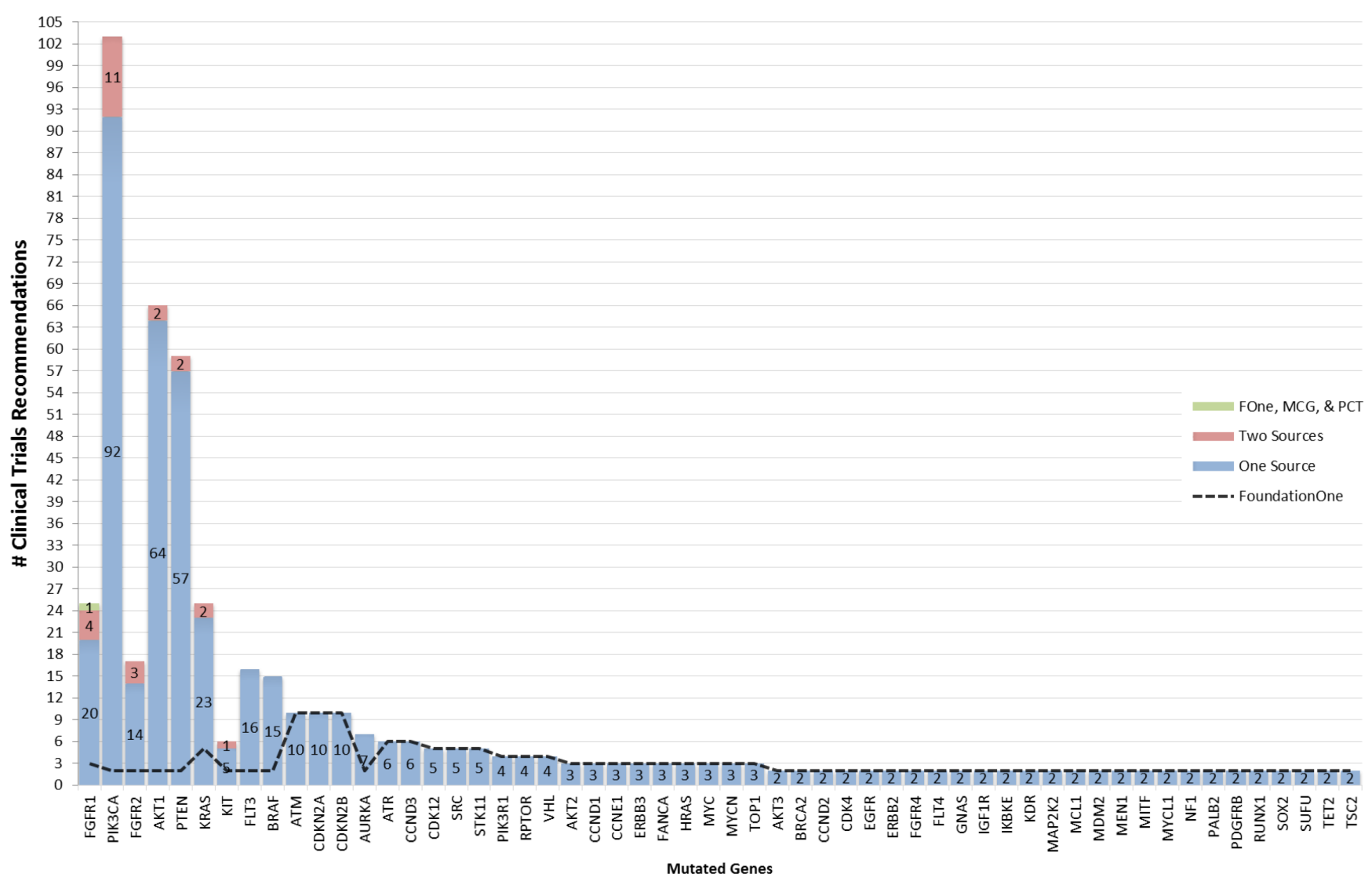

Figure 2: Occurrence of clinical trial recommendations. Mutated genes from highest to lowest number of sources are listed along $\mathrm{x}$-axis and number of clinical trials grouped by number of contributing sources are stacked along $\mathrm{y}$-axis. 
ponatinib, and two other tools identified palifermin (but not ponatinib) as potential options, among other drugs. Neither of these drugs represents a valid option. Ponatinib is a tyrosine kinase inhibitor that primarily targets BCRABL but also inhibits the FGFR receptor family; however, it has been withdrawn from the market due to serious toxicity concerns in 2013 [19]. Palifermin is a truncated recombinant keratinocyte growth factor (KGF), which mimics the actions of endogenous KGF by binding to and activating the fibroblast growth factor receptor $2 \mathrm{~b}$ (FGFR2b) [20]. There is no plausible biological hypothesis, or preclinical, data suggesting that palifermin would work as an anticancer drug for FGFR4-amplified cancers.

Since drug approval status and clinical trial options evolve rapidly as does our understanding of molecular pathways and drug targets, expert curation is currently still critical for the accuracy of the data. Unfortunately, human review of data elements is low throughput, time consuming and may also be of variable quality. The annotation databases also often lack information on the effect of gene level alterations (e.g. loss-of-function mutation vs amplification or gain-of-function mutations) on the biological pathway activity that is involved. A further difficulty arises from the presence of multiple mutations in different genes in the same cancer which may result in multiple different drug recommendations (Figure 3). This is consistent with the notion that ultimately combinations of targeted drugs will be required for successful therapy [21]. However, most of the drug combinations that would match the molecular abnormalities in a particular cancer have never been tested formally for safety in a Phase I trial.

In summary, we examined 4 different websites and a commercial service that were designed to link mutated genes to potential therapeutic options and found only partial overlap in the treatment options that were identified for the same genomic abnormalities. The highly curated sites contain more accurate information but only cover a very small number of genes, while the sites that are more broadly applicable are also more error prone and can include outdated information or link mutations to drugs that are not supported by strong evidence. Further improvements in search algorithms, data integration from multiple sources, and rapid and real-time interpretation of the peer-reviewed literature combined with artificial intelligence tools will be required for the development of broadly applicable
A Cohort level

75 Metastatic Breast Cancer Specimens

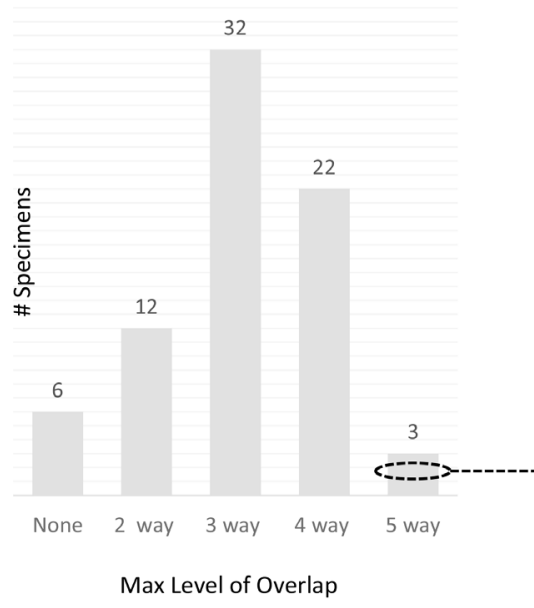

Figure 3: Overview of overlapping FDA approved drug recommendations at multiple levels. (A) 75 metastatic breast cancer specimens grouped by maximum level of overlapping FDA approved drug recommendation(s) for any of its mutated genes. (B) Venn diagram showing relationship between four recommendation sources (FoundationOne, Drug Gene Interaction Database, Personalized Cancer Therapy and cBioPortal) with mutated genes in sample specimen \# 37 [KIT, GNAS, KRAS, TOP1, AURKA, BRCA2, JAK2, SMAD4, TP53, ZNF217] based on maximum overlapping FDA approved drug recommendation(s). (C) Table listing all mutated genes for sample specimen \# 37 (column 1), their FDA approved drug recommendations (column 2), sources identified by green check mark (column 3-6) and a colored background that corresponds with maximum overlapping drug(s) represented in (B). 
software tools to assist therapeutic interpretation of high throughput genomic data $[22,23]$.

\section{MATERIALS AND METHODS}

\section{Patients and molecular data}

Molecular target profiling was performed in the context of a clinical study (clinicaltrial.gov: NCT01855503) to identify potentially actionable molecular abnormalities in prospectively collected metastatic tumor biopsy specimens of breast cancer ( $n=33$ liver, $n=13$ lymph node, $n=5$ breast, $n=5$ skin, $n=5$ soft tissue, $n=3$ lung, $n=3$ ovary, $n=8$ other). The study was approved by the Human Research Committee of the Yale Cancer Center. Seventy-five patients were accrued between June 2013 and June 2015. Target profiling was performed using the FoundationOne ${ }^{\mathrm{TM}}$ (Foundation Medicine Inc. Cambridge, MA) targeted sequencing assay that interrogates the coding sequence of 315 cancer-related genes and select introns from 28 genes often rearranged in solid tumors $[1,4]$. The assay report includes only molecular abnormalities that are deemed potentially actionable using a proprietary method and lists the drugs and clinical trials that represent therapeutic options (Supplementary Table S1 lists the specific abnormalities for each cases and the location of the biopsy).

\section{Web tools}

We ran the results from each case through 4 different websites that were designed to link mutated genes to potential therapeutic options. Table 3 presents an overview of the websites. The Drug-Gene Interaction Database (DGIdb) is affiliated with Washington University School of Medicine (http://dgidb.genome.wustl.edu) and integrates data from 13 primary sources to provide over 14,144 drug-gene interactions involving 2,611 genes and 6,307 drugs [13]. It's intended for researchers and has a specific disclaimer that the information is not to be used for medical advice. The input information is any gene symbol and the output is a list of generic, brand or developmental code names of drugs that are unfiltered for duplicates. The result also includes the predicted effect of the chemical entity on gene function (i.e. activator or inhibitor) and the name of the source database. This website does not identify clinical trials options. The My Cancer Genome (MCG) website is affiliated with Vanderbilt-Ingram Cancer Center (http://www. mycancergenome.org/) and provides extensive background information and potential clinical trial options for specific mutations in 55 genes in 21 different cancer types [14]. It is based on manual curation by physician-scientists and is intended to provide clinically relevant information for patients, and clinical researchers. During the query, the cancer type and gene is selected from a dropdown menu and clinical trial options are listed through the clinicaltrials.gov website using the NCT identifier number and study title. Specific drug recommendations are also provided since 2015. The Personalized Cancer Therapy (PCT) website is affiliated with MD Anderson Cancer Center (https://pct.mdanderson.org/). It includes detailed biological information in various disease contexts on 20 genes that can be selected from a dropdown menu. It relies both on manual curation and automatic database mining [15]. The intended audience is both patients and clinical researchers. Only clinically accessible drugs (approved or in active clinical trials) are listed and links to clinical trials are provided through the clinicaltrials. gov website. The cBioPortal (http://www.cbioportal. org/public-portal/) is affiliated with Memorial Sloan Kettering Cancer Center and provides access to a variety of information on 17,584 tumor samples from 69 cancer studies [16-18]. It integrates data from a large number of diverse sources and is intended audience are researchers. For a query, cancer data type and assay platform must be selected and a gene symbol entered. The output is presented as interactive molecular and epidemiologic data drawn from the selected database and data platform and can be accessed through various tabs which lead to tables and graphical results. Drugs that interact with the selected gene (either in preclinical or clinical experiments) can be found under the "network" tab and are embedded in an interactive gene network diagram; drug information can be filtered by FDA approval status. There is no listing of clinical trials. For this study, a gene was queried selecting the Breast cancer - TCGA (provisional) database and mutation and copy number level alterations, and potential drugs were identified through the network function.

\section{Data collection and analysis plan}

Treatment options were retrieved from all 4 web sites for all cases by entering every altered gene that was detected in a given cancer and reported by Foundation One. Abnormalities were collapsed at gene level because none of the sites were designed to interpret specific variants (with a few exception of canonical oncogenic mutations). Results were categorized as either FDA approved drugs (http://www.accessdata.fda.gov/scripts/ cder/drugsatfda/index.cfm) or clinical trial options defined by the National Clinical Trial (NCT) identifier. We use the term FDA-approved drug to indicate commercial availability under some cancer indication; we do not imply that a given drug is approved for breast cancer. We did not consider drugs or molecules that are not commercially available. Duplicate entries and drug name aliases were removed to create a single entry for each drug or trial. We assigned drug targets to "primary" or "secondary" categories whether the target represented the presumed main mechanism of action of a drug or an ancillary effect. The biopsies were collected over a 24 months period and 
Table 3: Comparison of 5 mutation based treatment recommendation sources

\begin{tabular}{|c|c|c|c|c|c|}
\hline Source & $\begin{array}{l}\text { FoundationOne }^{\circledR} \\
\text { www. } \\
\text { foundationone. } \\
\text { com }\end{array}$ & $\begin{array}{l}\text { My Cancer } \\
\text { Genome }^{\mathrm{TM}} \text { www. } \\
\text { mycancergenome.org }\end{array}$ & $\begin{array}{l}\text { Personalized } \\
\text { Cancer Therapy } \\
\text { pct.mdanderson. } \\
\text { org }\end{array}$ & $\begin{array}{l}\text { Drug Gene } \\
\text { Interaction } \\
\text { Database www. } \\
\text { dgidb.org }\end{array}$ & $\begin{array}{l}\text { cBioPortal www. } \\
\text { cbioportal.org }\end{array}$ \\
\hline Affiliation & commercial & $\begin{array}{l}\text { Vanderbilt-Ingram } \\
\text { Cancer Center }\end{array}$ & $\begin{array}{l}\text { MD Anderson } \\
\text { Cancer Center }\end{array}$ & $\begin{array}{c}\text { Washington } \\
\text { University School } \\
\text { of Medicine in St. } \\
\text { Louis }\end{array}$ & $\begin{array}{l}\text { Memorial Sloan } \\
\text { Kettering Cancer } \\
\text { Center }\end{array}$ \\
\hline Access & proprietary & free website & free website & free website & free website \\
\hline Intended Audience & $\begin{array}{l}\text { patients } \\
\text { clinicians }\end{array}$ & $\begin{array}{l}\text { patients } \\
\text { clinicians } \\
\text { researchers }\end{array}$ & $\begin{array}{l}\text { patients } \\
\text { clinicians }\end{array}$ & researchers only & researchers only \\
\hline Genes Covered & 315 & $55^{*}$ & 16 & $>2000$ & $>2000$ \\
\hline Data Entry & $\mathrm{n} / \mathrm{a}$ & dropdown list* & dropdown list & search box & search box \\
\hline Recommendations & $\begin{array}{l}\text { FDA approved } \\
\text { drugs } \\
\text { clinical trials }\end{array}$ & clinical trials & $\begin{array}{c}\text { FDA approved } \\
\text { drugs investigational } \\
\text { drugs clinical trials }\end{array}$ & $\begin{array}{l}\text { FDA approved drugs } \\
\text { investigational drugs } \\
\text { chemical compounds }\end{array}$ & $\begin{array}{l}\text { FDA approved } \\
\text { drugs } \\
\text { investigational } \\
\text { drugs chemical } \\
\text { compounds }\end{array}$ \\
\hline \multirow[t]{2}{*}{ Output } & $\begin{array}{l}\text { Drugs according } \\
\text { to mutated gene } \\
\text { with particular } \\
\text { variant are listed in } \\
\text { a table by generic } \\
\text { name based on } \\
\text { FDA approval } \\
\text { for patient's } \\
\text { malignancy } \\
\text { versus any other } \\
\text { malignancies. }\end{array}$ & $\begin{array}{l}\text { Drugs according to } \\
\text { mutated gene are } \\
\text { listed in a table by } \\
\text { generic, trade, code } \\
\text { and/or chemical name. }\end{array}$ & $\begin{array}{l}\text { Drugs according } \\
\text { to mutated gene } \\
\text { are listed in a } \\
\text { table by generic, } \\
\text { code or chemical } \\
\text { name along with } \\
\text { columns for } \\
\text { alternative names, } \\
\text { molecular targets, } \\
\text { FDA indications if } \\
\text { applicable and phase } \\
\text { of development. }\end{array}$ & \multirow{2}{*}{$\begin{array}{l}\text { Drugs according } \\
\text { to mutated gene } \\
\text { are listed in a } \\
\text { table unfiltered } \\
\text { for duplicates \& } \\
\text { unlabeled by FDA } \\
\text { status by generic, } \\
\text { brand, code or } \\
\text { chemical name } \\
\text { along with predicted } \\
\text { interaction and data } \\
\text { source. }\end{array}$} & \multirow{2}{*}{$\begin{array}{c}\text { Drugs according } \\
\text { to mutated gene } \\
\text { are each uniquely } \\
\text { distributed on an } \\
\text { interactive gene } \\
\text { network diagram } \\
\text { by a connecter } \\
\text { line that indicates } \\
\text { predicted level of } \\
\text { action on network } \\
\text { and FDA status } \\
\text { based on color of } \\
\text { connecter. }\end{array}$} \\
\hline & $\begin{array}{l}\text { Clinical trials } \\
\text { according to } \\
\text { mutated gene with } \\
\text { particular variant } \\
\text { are listed in a } \\
\text { table with study } \\
\text { title, trial phase, } \\
\text { available locations } \\
\text { and NCT identifier } \\
\text { number. }\end{array}$ & $\begin{array}{c}\text { Clinical trials } \\
\text { according to mutated } \\
\text { gene in specific } \\
\text { malignancy are listed } \\
\text { in a tabular format } \\
\text { subdivided by location } \\
\text { (US, International, } \\
\text { Unknown) with NCT } \\
\text { identifier number } \\
\text { study title \& trial } \\
\text { phase. }\end{array}$ & $\begin{array}{l}\text { Clinical trials } \\
\text { according to } \\
\text { mutated gene are } \\
\text { listed in a table } \\
\text { by NCT identifier } \\
\text { number and study } \\
\text { title along with } \\
\text { a link for more } \\
\text { information. }\end{array}$ & & \\
\hline Algorithm & proprietary & $\begin{array}{c}\text { Internal expert } \\
\text { curation of multiple } \\
\text { databases. }\end{array}$ & $\begin{array}{l}\text { Combination of } \\
\text { internal natural } \\
\text { language processing } \\
\text { code and internal } \\
\text { expert curation of } \\
\text { multiple databases. }\end{array}$ & $\begin{array}{l}\text { Combination of } \\
\text { open source code } \\
\text { \& internal expert } \\
\text { curation of both } \\
\text { externally curated } \\
\text { and uncurated } \\
\text { databases. }\end{array}$ & $\begin{array}{c}\text { Combination } \\
\text { of open source } \\
\text { code and internal } \\
\text { expert curation of } \\
\text { externally curated } \\
\text { databases and } 69 \\
\text { cancer datasets. }\end{array}$ \\
\hline $\begin{array}{l}\text { Background } \\
\text { Source }\end{array}$ & $\begin{array}{l}\text { brief description \& } \\
\text { primary reference } \\
\text { for drug provided }\end{array}$ & $\begin{array}{c}\text { background } \\
\text { information and } \\
\text { primary reference on } \\
\text { mutated gene provided }\end{array}$ & $\begin{array}{l}\text { brief description \& } \\
\text { primary reference } \\
\text { for drug provided }\end{array}$ & $\begin{array}{c}\text { name of drug } \\
\text { database provided }\end{array}$ & $\begin{array}{c}\text { name of drug } \\
\text { database provided }\end{array}$ \\
\hline Last update noted & $\mathrm{n} / \mathrm{a}$ & Yes & Yes & Yes & Yes \\
\hline
\end{tabular}

*Total number of genes listed under any 1 of 21 malignancies, excluding repeats. Note: Gene list \& access to information is restricted by required selection of malignancy. 
the drugs and clinical trial options reported in the results reflect the options that were available when the test was performed. To adjust for possible time-related discordance in treatment options, we reviewed each case for this possible bias. Websites were last accessed on August 7, 2015 .

\section{ACKNOWLEDGMENTS}

This study was supported by an award from the Breast Cancer Research Foundation to LP and molecular analysis of cancers were provided by Foundation Medicine through a research collaboration.

\section{CONFLICTS OF INTEREST}

No conflicts to disclose except for exceptions below:

Eric Reiner: Consulting or Advisory Role: BSCI, Surefire Medical; Other Relationship: Sirtex Medical.

Sarah Schellhorn Mougalian: Stock and Other Ownership Interests: Gilead Science, Coronado Biosciences, Roche; Research Funding: Genentech.

Maysa M. Abu-Khalaf: Research Funding: Novartis, Merck, Clovis Oncology, Pfizer, Genentech/ Roche, Merrimack.

Lajos Pusztai: Consulting or Advisory Role: Clovis Oncology, Celgene; Honoraria: BioTheranostics, Pfizer; Research Funding: Foundation Medicine, Merck, Genentech.

\section{REFERENCES}

1. Vasan N, Yelensky R, Wang K, Moulder S, Dzimitrowicz H, Avritscher R, Wang B, Wu Y, Cronin MT, Palmer G, Symmans WF, Miller VA, Stephens P, et al. A targeted next-generation sequencing assay detects a high frequency of therapeutically targetable alterations in primary and metastatic breast cancers: implications for clinical practice. Oncologist. 2014; 19:453-458.

2. McShane LM, Cavenagh MM, Lively TG, Eberhard DA, Bigbee WL, Williams PM, Mesirov JP, Polley M-YC, Kim KY, Tricoli JV, Taylor JMG, Shuman DJ, et al. Criteria for the use of omics-based predictors in clinical trials. Nature. 2013; 502:317-320.

3. Rehm HL, Bale SJ, Bayrak-Toydemir P, Berg JS, Brown KK, Deignan JL, Friez MJ, Funke BH, Hegde MR, Lyon E, Working Group of the American College of Medical G and Genomics Laboratory Quality Assurance C. ACMG clinical laboratory standards for next-generation sequencing. Genet Med. 2013; 15:733-747.

4. Frampton GM, Fichtenholtz A, Otto GA, Wang K, Downing SR, He J, Schnall-Levin M, White J, Sanford EM, An P, Sun J, Juhn F, Brennan K, et al. Development and validation of a clinical cancer genomic profiling test based on massively parallel DNA sequencing. Nat Biotechnol. 2013; 31:1023-1031.

5. Spencer DH, Tyagi M, Vallania F, Bredemeyer AJ, Pfeifer JD, Mitra RD, Duncavage EJ. Performance of Common Analysis Methods for Detecting Low-Frequency Single Nucleotide Variants in Targeted Next-Generation Sequence Data. The Journal of Molecular Diagnostics. $2014 ; 16: 75-88$.

6. Singh RR, Patel KP, Routbort MJ, Reddy NG, Barkoh BA, Handal B, Kanagal-Shamanna R, Greaves WO, Medeiros LJ, Aldape KD, Luthra R. Clinical validation of a next-generation sequencing screen for mutational hotspots in 46 cancer-related genes. J Mol Diagn. 2013; 15:607-622.

7. Gerlinger M, Rowan AJ, Horswell S, Larkin J, Endesfelder D, Gronroos E, Martinez P, Matthews N, Stewart A, Tarpey P, Varela I, Phillimore B, Begum S, et al. Intratumor Heterogeneity and Branched Evolution Revealed by Multiregion Sequencing. New England Journal of Medicine. 2012; 366:883-892.

8. Jones S, Anagnostou V, Lytle K, Parpart-Li S, Nesselbush M, Riley DR, Shukla M, Chesnick B, Kadan M, Papp E, Galens KG, Murphy D, Zhang T, et al. Personalized genomic analyses for cancer mutation discovery and interpretation. Science Translational Medicine. 2015; 7:283ra253-283ra253.

9. Kircher M, Witten DM, Jain P, O'Roak BJ, Cooper GM, Shendure J. A general framework for estimating the relative pathogenicity of human genetic variants. Nat Genet. 2014; 46:310-315.

10. Yang W, Soares J, Greninger P, Edelman EJ, Lightfoot H, Forbes S, Bindal N, Beare D, Smith JA, Thompson IR, Ramaswamy S, Futreal PA, Haber DA, et al. Genomics of Drug Sensitivity in Cancer (GDSC): a resource for therapeutic biomarker discovery in cancer cells. Nucleic Acids Res. 2013; 41:D955-961.

11. Jürgensmeier JM, Eder JP, Herbst RS. New strategies in personalized medicine for solid tumors: molecular markers and clinical trial designs. Clin Cancer Res. 2014; 20:4425-4435.

12. Meric-Bernstam F, Farhangfar C, Mendelsohn J, Mills GB. Building a personalized medicine infrastructure at a major cancer center. J Clin Oncol. 2013; 31:1849-1857.

13. Griffith M, Griffith OL, Coffman AC, Weible JV, McMichael JF, Spies NC, Koval J, Das I, Callaway MB, Eldred JM, Miller CA, Subramanian J, Govindan R, et al. DGIdb - Mining the druggable genome. Nat Methods. $2013 ; 10$.

14. Yeh P, Chen H, Andrews J, Naser R, Pao W, Horn L. DNA-Mutation Inventory to Refine and Enhance Cancer Treatment (DIRECT): a catalog of clinically relevant cancer mutations to enable genome-directed anticancer therapy. Clin Cancer Res. 2013; 19:1894-1901.

15. Knowledge Base for Precision Oncology. 
16. cBioPortal for Cancer Genomics: About Us.

17. Gao J, Aksoy BA, Dogrusoz U, Dresdner G, Gross B, Sumer SO, Sun Y, Jacobsen A, Sinha R, Larsson E, Cerami E, Sander C, Schultz N. Integrative analysis of complex cancer genomics and clinical profiles using the cBioPortal. Sci Signal. 2013; 6:pl1.

18. Cerami E, Gao J, Dogrusoz U, Gross BE, Sumer SO, Aksoy BA, Jacobsen A, Byrne CJ, Heuer ML, Larsson E, Antipin Y, Reva B, Goldberg AP, et al. The cBio cancer genomics portal: an open platform for exploring multidimensional cancer genomics data. Cancer Discov. 2012; 2:401-404.

19. Gainor JF, Chabner BA. Ponatinib: Accelerated Disapproval. Oncologist. 2015; 20:847-848.

20. Blijlevens N, Sonis S. Palifermin (recombinant keratinocyte growth factor-1): a pleiotropic growth factor with multiple biological activities in preventing chemotherapy- and radiotherapy-induced mucositis. Ann Oncol. 2007; 18:817-826.

21. Al-Lazikani B, Banerji U, Workman P. Combinatorial drug therapy for cancer in the post-genomic era. Nat Biotechnol. 2012; 30:679-692.

22. Meric-Bernstam F, Johnson A, Holla V, Bailey AM, Brusco L, Chen K, Routbort M, Patel KP, Zeng J, Kopetz S, Davies MA, Piha-Paul SA, Hong DS, et al. A decision support framework for genomically informed investigational cancer therapy. J Natl Cancer Inst. 2015; 107.

23. Ratner M. IBM's Watson Group signs up genomics partners. Nat Biotech. 2015; 33:10-11. 